\title{
ON THE PRICING OF CALL-PUT PARITIES OF ASIAN OPTIONS BY REDUCED DIFFERENTIAL TRANSFORM ALGORITHM
}

\section{JAVED HUSSAIN*, MUHAMMAD SHOAIB KHAN}

\author{
Sukkur IBA University, Pakistan \\ ${ }^{*}$ Corresponding author: javed.brohi@iba-suk.edu.pk
}

\begin{abstract}
The key aim of the paper is to show that how the efficiently the Reduced Differential Transform Algorithm (RDTA) can be employed to price the exotic financial options. In this paper we have computed the exact solution of the parabolic partial differential equation governing the dynamics of put-call parity in the mathematical theory of Asian options, by means of RDTA.
\end{abstract}

\section{Introduction}

An option is an agreement that allows the holder to buy (call option) or sell (put option) at a specified future time (expiration or maturity time) an underlying asset at a specified price (strike or exercise price).

Pricing of an Asian option is always complicated due to its path dependents derivatives whose payoffs depend on some form of averaged prices of the underlying asset and no closed form solution exist in general. Pricing them efficiently and accurately is very important both in theory and practice. Asian option can avoid manipulation of the stock near expiration time. Asian options are popular in the financial community as well as in the over-the-counter (OTC) market because they are often cheaper than the equivalent classical European options.

Several analytical approaches have been proposed to address the problem. In [?] a closed form solution of the no-arbitrage price of arithmetic averaged fixed strike price is obtained through the inversion of a Laplace Transform.

Received December $12^{\text {th }}, 2019$; accepted January $14^{\text {th }}, 2020$; published May $1^{\text {st }}, 2020$.

2010 Mathematics Subject Classification. 35Q91, 91G80.

Key words and phrases. Asian option; Put-Call Parity; Reduced Differential Transform Algorithm.

(C)2020 Authors retain the copyrights of their papers, and all open access articles are distributed under the terms of the Creative Commons Attribution License. 
The standard approximation methods based on partial differential equations require some regularity conditions of the solution of the no-arbitrage PDE. Considering path dependent contingent claims in general setting, the PDE is a strongly degenerate parabolic equation in three dimensions (time, the underlying asset price, and the path-dependent variable). In this setting, the needed regularity seemed out of reach. To avoid this difficulty, many authors considered a two-dimensional second-order PDE which is obtained from the original one through a change of variable (similarity reduction method) when the contingent claim final payoff has a particular form (see $[2,6,7,11,20,24,25]$ ). This method covers a large set of contingent claim contracts, including arithmetic Asin options, but not a contingent claim characterized by a general final payoff.

The Black-Scholes PDE for the fixed strike arithmetic Asian options, considering dividend (see, [17], pp. $277-278)$ is

$$
\begin{gathered}
\frac{\partial V}{\partial t}+\frac{S-J}{t} \frac{\partial V}{\partial J}+\frac{\sigma^{2}}{2} S^{2} \frac{\partial^{2} V}{\partial S^{2}}+(r-q) S \frac{\partial V}{\partial S}-r V=0 \\
V(S, J, T)=\left\{\begin{array}{ll}
(J-K)^{+}, & (\text {call option with fixed strike price) } \\
(K-J)^{+}, & \text {(put option with fixed strike price) }
\end{array} .\right.
\end{gathered}
$$

$V$ denote the price of an Asian option, $S$ is the value of the underlying asset, $K$ the exercise price, $T$ the expiration date, $r$ is the interest rate, $q$ is the continuously paid dividend, $\sigma$ is the asset volatility, and $J=\frac{1}{t} \int_{0}^{t} S_{\tau} d \tau$ be the path variable, denote the average of asset price for the period up to $t$.

It is well known that the difference between the prices of European vanilla call and put options is equal to a European forward contract. Similarly, we have put-call parity relations for European style Asian options [16].

Let $C_{f i x}(S, J, t)$ and $P_{f i x}(S, J, t)$ denote the price of the fixed strike arithmetic averaging Asian call option and put option, respectively. Their terminal payoff functions are given by

$$
\begin{aligned}
& C_{f i x}(S, J, T)=(J-K)^{+}=\max (J-K, 0) \\
& P_{f i x}(S, J, T)=(K-J)^{+}=\max (K-J, 0)
\end{aligned}
$$

Let $W(S, J, t)$ denote the difference of $C_{f i x}$ and $P_{f i x}$. Since both $C_{f i x}$ and $P_{f i x}$ are governed by the same differential equation [see (1.1)], so does $W(S, J, t)$. The terminal condition of their difference $W(S, J, t)$ is given by

$$
W(S, J, T)=(J-K)^{+}-(K-J)^{+}=J-K
$$


Hence the problem we have to investigate is

$$
\begin{gathered}
\frac{\partial W}{\partial t}+\frac{S-J}{t} \frac{\partial W}{\partial J}+\frac{\sigma^{2}}{2} S^{2} \frac{\partial^{2} W}{\partial S^{2}}+(r-q) S \frac{\partial W}{\partial S}-r W=0 \\
\left.W\right|_{t=T}=(J-K)^{+}-(K-J)^{+}=J-K
\end{gathered}
$$

In a similar manner, we have other PDEs of put-call parities of other types of Asian options (floating/fixed and arithmetic/geometric averaging) for the the investigation will see in section 3 .

In this article, the fundamental objective is to investigate all four versions of PDEs of put-call parities of Asian options using Reduced Differential Transform Method(RDTM) to validate the method. RDTM proposed by Keskin [12] and successfully employed to solve many types of linear and nonlinear PDEs. RDTM is a reliable semi-analytic method subject to appropriate initial condition. Taking into consideration of this method, it is possible to find an exact solution or a closed approximate solution of a differential equation.

\section{Reduced Differential Transform Method}

Consider a function of two variables $u(x, t)$ and suppose that it can be represented as a product of two single-variable functions, i.e., $u(x, t)=f(x) g(t)$. Based on the properties of one dimensional differential transform, the function $u(x, t)$ can be represented as follows:

$$
u(x, t)=\left(\sum_{i=0}^{\infty} F(i) x^{i}\right)\left(\sum_{i=0}^{\infty} G(j) t^{j}\right)=\sum_{k=0}^{\infty} U_{k}(x) t^{k},
$$

where $U_{k}(x)$ is called $t$-dimensional spectrum function of $u(x, t)$. The basic definitions of RDTM are introduced as follows (cf. [12-15]):

Definition 2.1. If function $u(x, t)$ is analytic and differentiated continuously with respect to time $t$ and space $x$ in the domain of interest, then let

$$
U_{k}(x)=\frac{1}{k !}\left[\frac{\partial^{k}}{\partial t^{k}} u(x, t)\right]_{t=0}
$$

where the $t$-dimensional spectrum function $U_{k}(x)$ is the transformed function. In this paper, the lowercase $u(x, t)$ represents the original function, while the uppercase $U_{k}(x)$ stands for the transformed function.

Definition 2.2. The differential inverse transform of $U_{k}(x)$ is defined as follows:

$$
u(x, t)=\sum_{k=0}^{\infty} U_{k}(x) t^{k}
$$

Then, combining Eqs. (2.2) and (2.3) we write

$$
u(x, t)=\sum_{k=0}^{\infty} \frac{1}{k !}\left[\frac{\partial^{k}}{\partial t^{k}} u(x, t)\right]_{t=0} t^{k}
$$


from the above definitions, it can be found that the concept of the RDTM is derived from the power series expansion.

To illustrate the basic concepts of the RDTM, consider the following nonlinear partial differential equation written in an operator form

$$
L u(x, t)+R u(x, t)+N u(x, t)=g(x, t),
$$

with initial condition $u(x, 0)=f(x)$, where $L=\frac{\partial}{\partial t}, R$ is a linear operator which has partial derivatives, $N u(x, t)$ is a nonlinear operator and $g(x, t)$ is an in-homogeneous term. According to the RDTM, we can construct the following iteration formula:

$$
(k+1) U_{k+1}(x)=G_{k}(x)-R U_{k}(x)-N U_{k}(x),
$$

where $U_{k}(x), R U_{k}(x), N U_{k}(x)$ and $G_{k}(x)$ are the transformations of the functions $L u(x, t), R u(x, t)$, $N u(x, t)$ and $g(x, t)$ respectively.

From the initial condition, we write

$$
U_{0}(x)=f(x) .
$$

Substituting (2.7) into (2.6) and by a straightforward iterative calculation, we get the following $U_{k}(x)$ values. Then the inverse transformation of the set of values $\left\{U_{k}(x)\right\}_{k=0}^{n}$ gives approximation solutions as,

$$
\widetilde{u}_{n}(x, t)=\sum_{k=0}^{n} U_{k}(x) t^{k}
$$

where $n$ is the order of approximation solution.

Therefore, the exact solution of problems given by

$$
u(x, t)=\lim _{n \rightarrow \infty} \widetilde{u}_{n}(x, t)
$$

The fundamental mathematical operations performed by RDTM can be readily obtained and are listed in following table.

Table 1. Reduced Differential Transformation

$$
\left[\begin{array}{ll}
\text { Functional Form } & \text { Transformed Form } \\
u(x, t) & U_{k}(x)=\frac{1}{k !}\left[\frac{\partial^{k}}{\partial t^{k}} u(x, t)\right]_{t=0} \\
w(x, t)=u(x, t) \pm v(x, t) & W_{k}(x)=U_{k}(x) \pm V_{k}(x) \\
w(x, t)=\alpha u(x, t) & W_{k}(x)=\alpha U_{k}(x), \alpha \text { is a constant } \\
w(x, t)=x^{m} t^{n} & W_{k}(x)=x^{m} \delta(k-n), \text { the Kronecker delta } \\
w(x, t)=x^{m} t^{n} u(x, t) & W_{k}(x)=x^{m} U_{k-n}(x), \text { when } k \geq n \text { else } 0 . \\
w(x, t)=u(x, t) v(x, t) & W_{k}(x)=\sum_{r=0}^{k} V_{r}(x) U_{k-r}(x)=\sum_{r=0}^{k} U_{r}(x) V_{k-r}(x) \\
w(x, t)=\frac{\partial^{r}}{\partial t^{r}} u(x, t) & W_{k}(x)=(k+1) \ldots(k+r) U_{k+r}(x)=\frac{(k+r) !}{k !} U_{k+r}(x) \\
w(x, t)=\frac{\partial}{\partial x} u(x, t) & W_{k}(x)=\frac{\partial}{\partial x} U_{k}(x)
\end{array}\right]
$$




\section{Pricing of Four Versions of Call-Put Parities of Asian Options}

In this section, we will price call-put parities standard PDEs of four version of Asian options [17], that are:

1) Arithmetic average Asian option with fixed strike price.

2) Geometric average Asian option with fixed strike price.

3) Arithmetic average Asian option with floating strike price.

4) Geometric average Asian option with floating strike price.

by Reduced Differential Transform Method to validate the efficiency of the RDTM.

\subsection{Call-Put Parity for Arithmetic Average Asian Option with Fixed Strike Price.}

Assume that $C(S, J, t)$ and $P(S, J, t)$ denote the valuation of an Asian call and put option, respectively. define

$$
W(S, J, t)=C(S, J, t)-P(S, J, t)
$$

Then in $\{0 \leq S<\infty, 0 \leq J<\infty, 0 \leq t \leq T\}, W$ satisfies

$$
\begin{gathered}
\frac{\partial W}{\partial t}+\frac{S-J}{t} \frac{\partial W}{\partial J}+\frac{\sigma^{2}}{2} S^{2} \frac{\partial^{2} W}{\partial S^{2}}+(r-q) S \frac{\partial W}{\partial S}-r W=0 \\
\left.W\right|_{t=T}=(J-K)^{+}-(K-J)^{+}=J-K .
\end{gathered}
$$

By the use of change of variable,

$$
\xi=\frac{T K-t J}{S}
$$

the function

$$
w=\frac{T}{S} W
$$

satisfies the Cauchy problem [17] in the domain $\{\xi \in \mathbb{R}, 0 \leq t \leq T\}$ :

$$
\begin{gathered}
\frac{\partial w}{\partial t}+\frac{\sigma^{2}}{2} \xi^{2} \frac{\partial^{2} w}{\partial \xi^{2}}-[(r-q) \xi+1] \frac{\partial w}{\partial \xi}-q w=0, \\
w(\xi, T)=-\xi
\end{gathered}
$$

where

$$
w=w(\xi, t)
$$

For convenience to apply RDTM, we are assuming that $w(\xi, t)=u(\xi, t)$, Thus

$$
\begin{gathered}
\frac{\partial u}{\partial t}+\frac{\sigma^{2}}{2} \xi^{2} \frac{\partial^{2} u}{\partial \xi^{2}}-[(r-q) \xi+1] \frac{\partial u}{\partial \xi}-q u=0, \\
\operatorname{with} u(\xi, T)=-\xi .
\end{gathered}
$$

We take the change of variable $\tau=T-t$, to convert terminal condition into initial condition, so

$$
\frac{\partial u}{\partial t}=-\frac{\partial u}{\partial \tau}
$$


or

$$
\begin{gathered}
\frac{\partial u}{\partial \tau}=\frac{\sigma^{2}}{2} \xi^{2} \frac{\partial^{2} u}{\partial \xi^{2}}-[(r-q) \xi+1] \frac{\partial u}{\partial \xi}-q u \\
\left.\operatorname{with} u\right|_{\tau=0}=-\xi
\end{gathered}
$$

where

$$
u=u(\xi, \tau)
$$

According to the RDTM, we construct the following recurrence relation for the Eq. (3.3)

$$
(m+1) U_{m+1}(\xi)=\frac{\sigma^{2}}{2} \xi^{2} \frac{\partial^{2}}{\partial \xi^{2}} U_{m}(\xi)-[(r-q) \xi+1] \frac{\partial}{\partial \xi} U_{m}(\xi)-q U_{m}(\xi)
$$

Note: $u=u(\xi, \tau) \rightarrow$ functional form and $U_{m}=U_{m}(\xi) \rightarrow$ transformed form.

For $m=0$

$$
U_{1}(\xi)=\frac{\sigma^{2}}{2} \xi^{2} \frac{\partial^{2}}{\partial \xi^{2}} U_{0}(\xi)-[(r-q) \xi+1] \frac{\partial}{\partial \xi} U_{0}(\xi)-q U_{0}(\xi)
$$

From the initial condition, we write

$$
u(\xi, 0)=U_{0}(\xi)=-\xi
$$

So

$$
\frac{\partial}{\partial \xi} U_{0}(\xi)=-1, \quad \frac{\partial^{2}}{\partial \xi^{2}} U_{0}(\xi)=0
$$

Thus

$$
U_{1}(\xi)=1+r \xi
$$

For $m=1$

$$
2 U_{2}(\xi)=\frac{\sigma^{2}}{2} \xi^{2} \frac{\partial^{2}}{\partial \xi^{2}} U_{1}(\xi)-[(r-q) \xi+1] \frac{\partial}{\partial \xi} U_{1}(\xi)-q U_{1}(\xi)
$$

we get,

$$
U_{2}(\xi)=-\frac{1}{2}\left[r^{2} \xi+\frac{r^{2}-q^{2}}{r-q}\right]
$$

For $m=2$

$$
3 U_{3}(\xi)=\frac{\sigma^{2}}{2} \xi^{2} \frac{\partial^{2}}{\partial \xi^{2}} U_{2}(\xi)-[(r-q) \xi+1] \frac{\partial}{\partial \xi} U_{2}(\xi)-q U_{2}(\xi)
$$

we have,

$$
U_{3}(\xi)=\frac{1}{3 !}\left[r^{3} \xi+\frac{r^{3}-q^{3}}{r-q}\right]
$$

By an inductive argument we have following,

$$
U_{m}(\xi)=\frac{(-1)^{m+1}}{m !}\left[r^{m} \xi+\frac{r^{m}-q^{m}}{r-q}\right], \text { where } m \geq 0 .
$$


For the solution, differential inverse transform of $U_{m}(\xi)$ is defined as below:

$$
\begin{aligned}
& u(\xi, \tau)=\sum_{m=0}^{\infty} U_{m}(\xi) \tau^{m} \\
& u(\xi, \tau)=\sum_{m=0}^{\infty} \frac{(-1)^{m+1} \tau^{m}}{m !}\left[r^{m} \xi+\frac{r^{m}-q^{m}}{r-q}\right] \\
& u(\xi, \tau)=\sum_{m=0}^{\infty} \frac{(-1)^{m+1} \tau^{m}}{m !} r^{m} \xi+\sum_{m=0}^{\infty} \frac{(-1)^{m+1} \tau^{m}}{m !} \frac{r^{m}-q^{m}}{r-q} \\
& u(\xi, \tau)=-\xi \sum_{m=0}^{\infty} \frac{(-1)^{m} \tau^{m} r^{m}}{m !}-\frac{1}{r-q} \sum_{m=0}^{\infty} \frac{(-1)^{m} \tau^{m}}{m !}\left(r^{m}-q^{m}\right) \\
& u(\xi, \tau)=-\xi \sum_{m=0}^{\infty} \frac{(-r \tau)^{m}}{m !}-\frac{1}{r-q}\left[\sum_{m=0}^{\infty} \frac{(-r \tau)^{m}}{m !}-\sum_{m=0}^{\infty} \frac{(-q \tau)^{m}}{m !}\right] \\
& u(\xi, \tau)=-\xi e^{-r \tau}-\frac{1}{r-q}\left[e^{-r \tau}-e^{-q \tau}\right]
\end{aligned}
$$

Here

$$
\xi=\frac{T K-t J}{S}, u(\xi, t)=w(\xi, t)
$$

where $\tau$ in terms of $t$ so above equation can be written as

$$
\begin{gathered}
w(\xi, t)=-\frac{T K-t J}{S} e^{-r(T-t)}-\frac{1}{r-q}\left[e^{-r(T-t)}-e^{-q(T-t)}\right] \\
\because w(\xi, t)=\frac{T}{S} W \Rightarrow W=\frac{S}{T} w(\xi, t)
\end{gathered}
$$

The exact solution, in closed form, is given by

$$
\begin{aligned}
C(S, J, t)-P(S, J, t) & =W(S, J, t) \\
W(S, J, t) & =\left[\frac{t}{T} J-\frac{S}{(r-q) T}-K\right] e^{-r(T-t)}-\frac{S}{(r-q) T} e^{-q(T-t)}
\end{aligned}
$$

which is same solution as obtained in [17]. Consider a six-month call option on stock. If $S_{0}=K=$ $\$ 145, r=6, q=3$ and $\sigma=29.5$ then graphs of $W(S, J, t)$, for average stock price $J$ ranging from $\$ 140$ to $\$ 150$, can be found in Figure 1 on next page.

\subsection{Call-Put Parity for Arithmetic Average Asian Option with Floating Strike Price.}

Consider

$$
W(S, J, t)=C(S, J, t)-P(S, J, t)
$$

then under appropriate transformations, standard call-put parities satisfies the Cauchy problem [17] in the domain $\{0 \leq \xi<\infty, 0 \leq t \leq T\}$ :

$$
\begin{gathered}
\frac{\partial w}{\partial t}+\frac{\sigma^{2}}{2} \xi^{2} \frac{\partial^{2} w}{\partial \xi^{2}}+[1-(r-q) \xi] \frac{\partial w}{\partial \xi}-q w=0 \\
\left.w\right|_{t=T}=1-\frac{\xi}{T}
\end{gathered}
$$




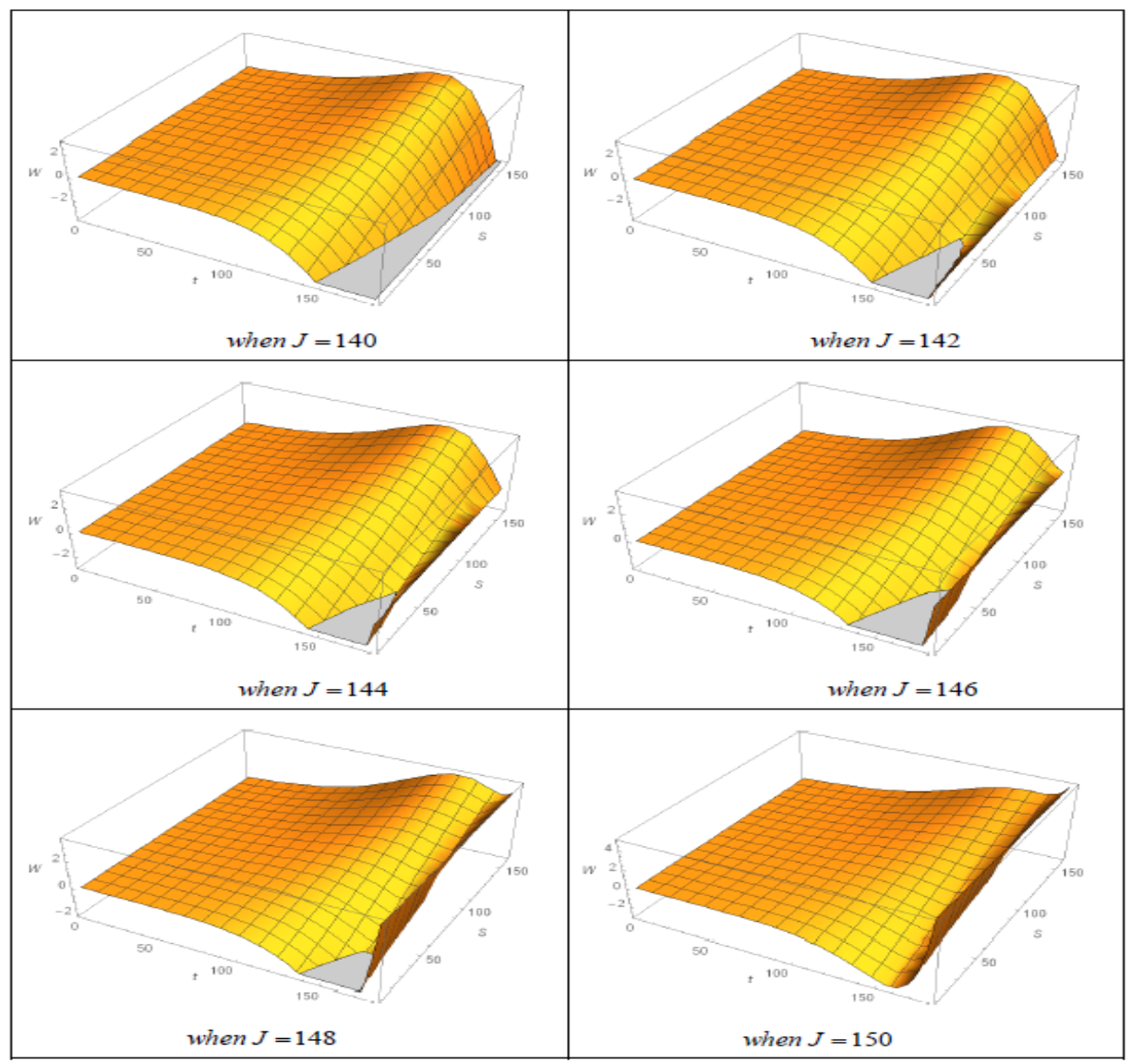

Figure 1. Plot of $\mathrm{W}(\mathrm{S}, \mathrm{J}, \mathrm{t})$ when $S_{0}=K=\$ 145, r=6, q=3$ and $\sigma=29.5$

where

$$
w=w(\xi, t)
$$

Taking into consideration $w(\xi, t)=u(\xi, t)$, for the sake of easy utility of RDTM, we have

$$
\begin{gathered}
\frac{\partial u}{\partial t}+\frac{\sigma^{2}}{2} \xi^{2} \frac{\partial^{2} u}{\partial \xi^{2}}+[1-(r-q) \xi] \frac{\partial u}{\partial \xi}-q u=0 \\
u(\xi, T)=1-\frac{\xi}{T}
\end{gathered}
$$

We take the change of variable $\tau=T-t$, to convert terminal condition into initial condition, we have

$$
\begin{gathered}
\frac{\partial u}{\partial \tau}=\frac{\sigma^{2}}{2} \xi^{2} \frac{\partial^{2} u}{\partial \xi^{2}}+[1-(r-q) \xi] \frac{\partial u}{\partial \xi}-q u \\
\left.u\right|_{\tau=0}=1-\frac{\xi}{T}
\end{gathered}
$$

Applying the RDTM to Eq. (3.5), we obtain the following recurrence equation

$$
(m+1) U_{m+1}(\xi)=\frac{\sigma^{2}}{2} \xi^{2} \frac{\partial^{2}}{\partial \xi^{2}} U_{m}(\xi)+[1-(r-q) \xi] \frac{\partial}{\partial \xi} U_{m}(\xi)-q U_{m}(\xi)
$$


For $m=0$

$$
U_{1}(\xi)=\frac{\sigma^{2}}{2} \xi^{2} \frac{\partial^{2}}{\partial \xi^{2}} U_{0}(\xi)+[1-(r-q) \xi] \frac{\partial}{\partial \xi} U_{0}(\xi)-q U_{0}(\xi)
$$

According to the initial condition, we can write

$$
u(\xi, 0)=U_{0}(\xi)=1-\frac{\xi}{T}
$$

So

$$
U_{1}(\xi)=\frac{1}{T}(r \xi-1)-q
$$

For $m=1$

$$
2 U_{2}(\xi)=\frac{\sigma^{2}}{2} \xi^{2} \frac{\partial^{2}}{\partial \xi^{2}} U_{1}(\xi)+[1-(r-q) \xi] \frac{\partial}{\partial \xi} U_{1}(\xi)-q U_{1}(\xi)
$$

having

$$
U_{2}(\xi)=-\frac{1}{2 !}\left[\frac{1}{T}\left\{r^{2} \xi-\frac{r^{2}-q^{2}}{r-q}\right\}-q^{2}\right]
$$

For $m=2$

$$
3 U_{3}(\xi)=\frac{\sigma^{2}}{2} \xi^{2} \frac{\partial^{2}}{\partial \xi^{2}} U_{2}(\xi)+[1-(r-q) \xi] \frac{\partial}{\partial \xi} U_{2}(\xi)-q U_{2}(\xi)
$$

we will obtained,

$$
U_{3}(\xi)=\frac{1}{3 !}\left[\frac{1}{T}\left\{r^{3} \xi-\frac{r^{3}-q^{3}}{r-q}\right\}-q^{3}\right]
$$

By an inductive argument we have following,

$$
U_{m}(\xi)=\frac{(-1)^{m+1}}{m !}\left[\frac{1}{T}\left\{r^{m} \xi-\frac{r^{m}-q^{m}}{r-q}\right\}-q^{m}\right], \text { Form } \geq 0
$$

For the solution, inverse differential transform of $U_{m}(\xi)$ is defined as below:

$$
\begin{aligned}
u(\xi, \tau) & =\sum_{m=0}^{\infty} U_{m}(\xi) \tau^{m} \\
u(\xi, \tau) & =\sum_{m=0}^{\infty} \frac{(-1)^{m+1}}{m !}\left[\frac{1}{T}\left\{r^{m} \xi-\frac{r^{m}-q^{m}}{r-q}\right\}-q^{m}\right] \tau^{m} \\
u(\xi, \tau) & =-\frac{\xi}{T} \sum_{m=0}^{\infty} \frac{(-r \tau)^{m}}{m !}+\frac{1}{T(r-q)} \sum_{m=0}^{\infty} \frac{(-r \tau)^{m}-(-q \tau)^{m}}{m !}+\sum_{m=0}^{\infty} \frac{(-q \tau)^{m}}{m !} \\
u(\xi, \tau) & =-\frac{\xi}{T} e^{-r \tau}+\frac{1}{T(r-q)}\left(e^{-r \tau}-e^{-q \tau}\right)+e^{-q \tau} \\
u(\xi, \tau) & =-\frac{\xi}{T} e^{-r \tau}+\frac{1}{T(r-q)} e^{-r \tau}\left(1-\frac{1}{T(r-q)}\right) e^{-q \tau}
\end{aligned}
$$

Here

$$
\xi=\frac{t J}{S}, u(\xi, t)=w(\xi, t)
$$

where $\tau$ in terms of $t$ so above equation can be written as

$$
\begin{gathered}
w(\xi, t)=-\frac{t J}{S T} e^{-r(T-t)}+\frac{1}{T(r-q)} e^{-r(T-t)}\left(1-\frac{1}{T(r-q)}\right) e^{-q(T-t)} \\
\because w=\frac{W}{S} \Rightarrow W(S, J, t)=S w(\xi, t)
\end{gathered}
$$


In closed form, the exact solution is given by

$$
C(S, J, t)-P(S, J, t)=-\frac{t}{T} J e^{-r(T-t)}+\frac{S}{T(r-q)} e^{-r(T-t)}\left(1-\frac{1}{T(r-q)}\right) S e^{-q(T-t)}
$$

which is same as obtained in [17]. Consider a six-month call option on stock. If $S_{0}=K=\$ 145, r=$ $6, q=3$ and $\sigma=29.5$ then graphs of $W(S, J, t)$, for average stock price $J$ ranging from $\$ 140$ to $\$ 150$, can be found in Figure 2.

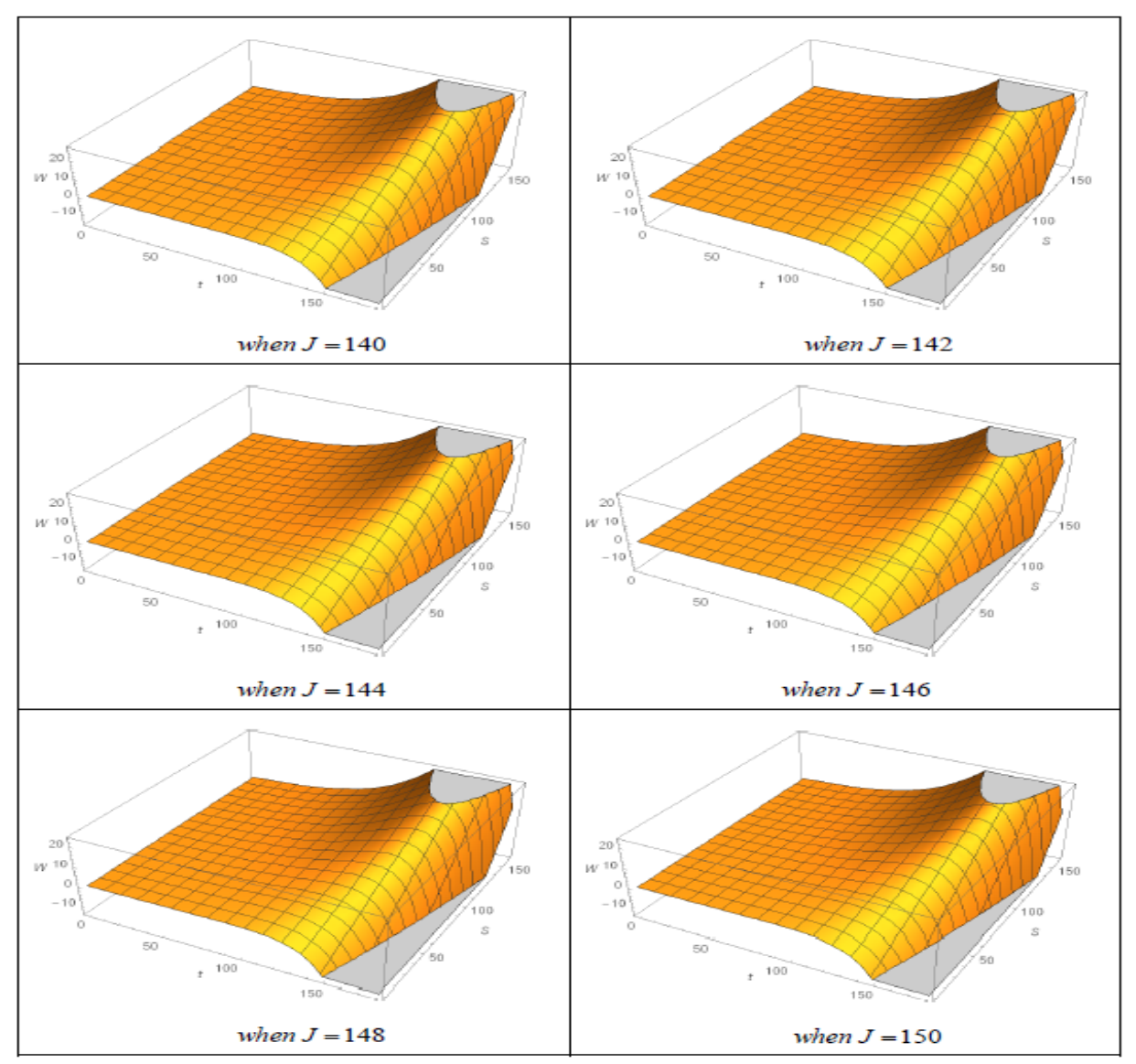

Figure 2. Plot of $\mathrm{w}(\mathrm{S}, \mathrm{J}, \mathrm{t})$ when $S_{0}=K=\$ 145, r=6, q=3$ and $\sigma=29.5$

\subsection{Call-Put Parity for Geometric Average Asian Option with Fixed Strike Price.}

Suppose that

$$
W(S, J, t)=C(S, J, t)-P(S, J, t)
$$

Thus in $\{0 \leq S<\infty, 0 \leq J<\infty, 0 \leq t \leq T\}, W$ satisfies the problem:

$$
\begin{gathered}
\frac{\partial W}{\partial t}+J \frac{\ln S-\ln J}{t} \frac{\partial W}{\partial J}+\frac{\sigma^{2}}{2} S^{2} \frac{\partial^{2} W}{\partial S^{2}}+(r-q) S \frac{\partial W}{\partial S}-r W=0 \\
\left.W\right|_{t=T}=(J-K)^{+}-(K-J)^{+}=J-K .
\end{gathered}
$$


By the transformation,

$$
\xi=\frac{t \ln J+(T-t) \ln S}{T}
$$

in $\{\xi \in \mathbb{R}, 0 \leq t \leq T\}, W$ satisfies [17]:

$$
\begin{gathered}
\frac{\partial W}{\partial t}+\frac{\sigma^{2}}{2}\left(\frac{T-t}{T}\right)^{2} \frac{\partial^{2} W}{\partial \xi^{2}}+\left(r-q-\frac{\sigma^{2}}{2}\right)\left(\frac{T-t}{T}\right) \frac{\partial W}{\partial \xi}-r W=0, \\
\left.W\right|_{t=T}=e^{\xi}-K .
\end{gathered}
$$

where

$$
W=W(\xi, t)
$$

For convenience, we are assuming that $W(\xi, t)=u(\xi, t)$, we get

$$
\begin{gathered}
\frac{\partial u}{\partial t}+\frac{\sigma^{2}}{2}\left(\frac{T-t}{T}\right)^{2} \frac{\partial^{2} u}{\partial \xi^{2}}+\left(r-q-\frac{\sigma^{2}}{2}\right)\left(\frac{T-t}{T}\right) \frac{\partial u}{\partial \xi}-r u=0, \\
\left.u\right|_{t=T}=e^{\xi}-K
\end{gathered}
$$

We are taking the change of variable $\tau=T-t$, we have

$$
\begin{gathered}
\frac{\partial u}{\partial \tau}=\frac{\sigma^{2}}{2}\left(\frac{\tau}{T}\right)^{2} \frac{\partial^{2} u}{\partial \xi^{2}}+\left(r-q-\frac{\sigma^{2}}{2}\right)\left(\frac{\tau}{T}\right) \frac{\partial u}{\partial \xi}-r u, \\
\left.u\right|_{\tau=0}=e^{\xi}-K .
\end{gathered}
$$

where

$$
u=u(\xi, \tau)
$$

By applying the RDTM, we construct the following iteration formula to Eq. (3.9)

$$
(m+1) U_{m+1}(\xi)=\frac{\sigma^{2}}{2 T^{2}} \frac{\partial^{2}}{\partial \xi^{2}} U_{m-2}(\xi)+\left(\frac{r-q-\frac{\sigma^{2}}{2}}{T}\right) \frac{\partial}{\partial \xi} U_{m-1}(\xi)-r U_{m}(\xi)
$$

Here we used the property if $v(x, t)=x^{m} t^{n} u(x, t)$ then $V_{k}(x)=x^{m} U_{k-n}(x)$ when $k \geq n$,else 0 , on first and second term.

By considering the initial condition, we can write

$$
U_{0}(\xi)=e^{\xi}-K
$$

For $m=0$

$$
U_{1}(\xi)=-r\left(e^{\xi}-K\right)
$$

For $m=1$

$$
U_{2}(\xi)=\frac{1}{2 !}\left\{\left(\frac{r-q-\frac{\sigma^{2}}{2}}{T}\right) e^{\xi}+r^{2}\left(e^{\xi}-K\right)\right\}
$$

For $m=2$

$$
3 U_{3}(\xi)=\frac{\sigma^{2}}{2 T^{2}} \frac{\partial^{2}}{\partial \xi^{2}} U_{0}(\xi)+\left(\frac{r-q-\frac{\sigma^{2}}{2}}{T}\right) \frac{\partial}{\partial \xi} U_{1}(\xi)-r U_{2}(\xi)
$$


we get,

$$
U_{3}(\xi)=\frac{1}{3 !}\left\{\frac{\sigma^{2}}{T^{2}} e^{\xi}-3 r e^{\xi}\left(\frac{r-q-\frac{\sigma^{2}}{2}}{T}\right)-r^{3}\left(e^{\xi}-K\right)\right\}
$$

For $m=3$

$$
4 U_{4}(\xi)=\frac{\sigma^{2}}{2 T^{2}} \frac{\partial^{2}}{\partial \xi^{2}} U_{1}(\xi)+\left(\frac{r-q-\frac{\sigma^{2}}{2}}{T}\right) \frac{\partial}{\partial \xi} U_{2}(\xi)-r U_{3}(\xi)
$$

Substitute $U_{1}(\xi), U_{2}(\xi)$ and $U_{3}(\xi)$ in above equation, we get

$$
U_{4}(\xi)=\frac{1}{4 !}\left\{-4 \frac{\sigma^{2}}{T^{2}} r e^{\xi}+3\left(\frac{r-q-\frac{\sigma^{2}}{2}}{T}\right)^{2} e^{\xi}+6\left(\frac{r-q-\frac{\sigma^{2}}{2}}{T}\right) r^{2} e^{\xi}+r^{4}\left(e^{\xi}-K\right)\right\}
$$

and so on.

For the solution differential inverse transform of $U_{m}(\xi)$ is defined as below:

$$
\begin{gathered}
u(\xi, \tau)=\sum_{m=0}^{\infty} U_{m}(\xi) \tau^{m} \\
u(\xi, \tau)=e^{\xi} e^{\left(\frac{\sigma^{2} \tau^{3}}{6 T^{2}}+\frac{\left(r-q-\frac{\sigma^{2}}{2}\right) \tau^{2}}{2 T}-r \tau\right)}-K e^{-r \tau} \\
\because \tau=T-t
\end{gathered}
$$

and from

$$
\xi=\frac{t \ln J+(T-t) \ln S}{T}
$$

we can write

$$
e^{\xi}=J^{\frac{t}{T}} S^{\frac{(T-t)}{T}}
$$

So finally, we have the exact solution, in closed form

$$
W(S, J, t)=\left\{J^{\frac{t}{T}} S^{\frac{(T-t)}{T}} e^{(T-t)\left(\frac{\sigma^{2}}{6}\left(\frac{T-t}{T}\right)^{2}+\frac{\left(r-q-\frac{\sigma^{2}}{2}\right)}{2}\left(\frac{T-t}{T}\right)\right)}-K\right\} e^{-r(T-t)}
$$

which is the same result as obtained in [17]. Consider a six-month call option on stock. If $S_{0}=K=$ $\$ 145, r=6, q=3$ and $\sigma=29.5$ then graphs of $W(S, J, t)$, for average stock price $J$ ranging from $\$ 140$ to $\$ 150$, can be found in Figure 3 on next page.

\subsection{Call-Put Parity for Geometric Average Asian Option with Floating Strike Price.}

Let

$$
W(S, J, t)=C(S, J, t)-P(S, J, t)
$$

Thus in $\{0 \leq S<\infty, 0 \leq J<\infty, 0 \leq t \leq T\}, W$ satisfies

$$
\begin{gathered}
\frac{\partial W}{\partial t}+J \frac{\ln S-\ln J}{t} \frac{\partial W}{\partial J}+\frac{\sigma^{2}}{2} S^{2} \frac{\partial^{2} W}{\partial S^{2}}+(r-q) S \frac{\partial W}{\partial S}-r W=0, \\
\left.W\right|_{t=T}=(S-J)^{+}-(J-S)^{+}=S-J .
\end{gathered}
$$




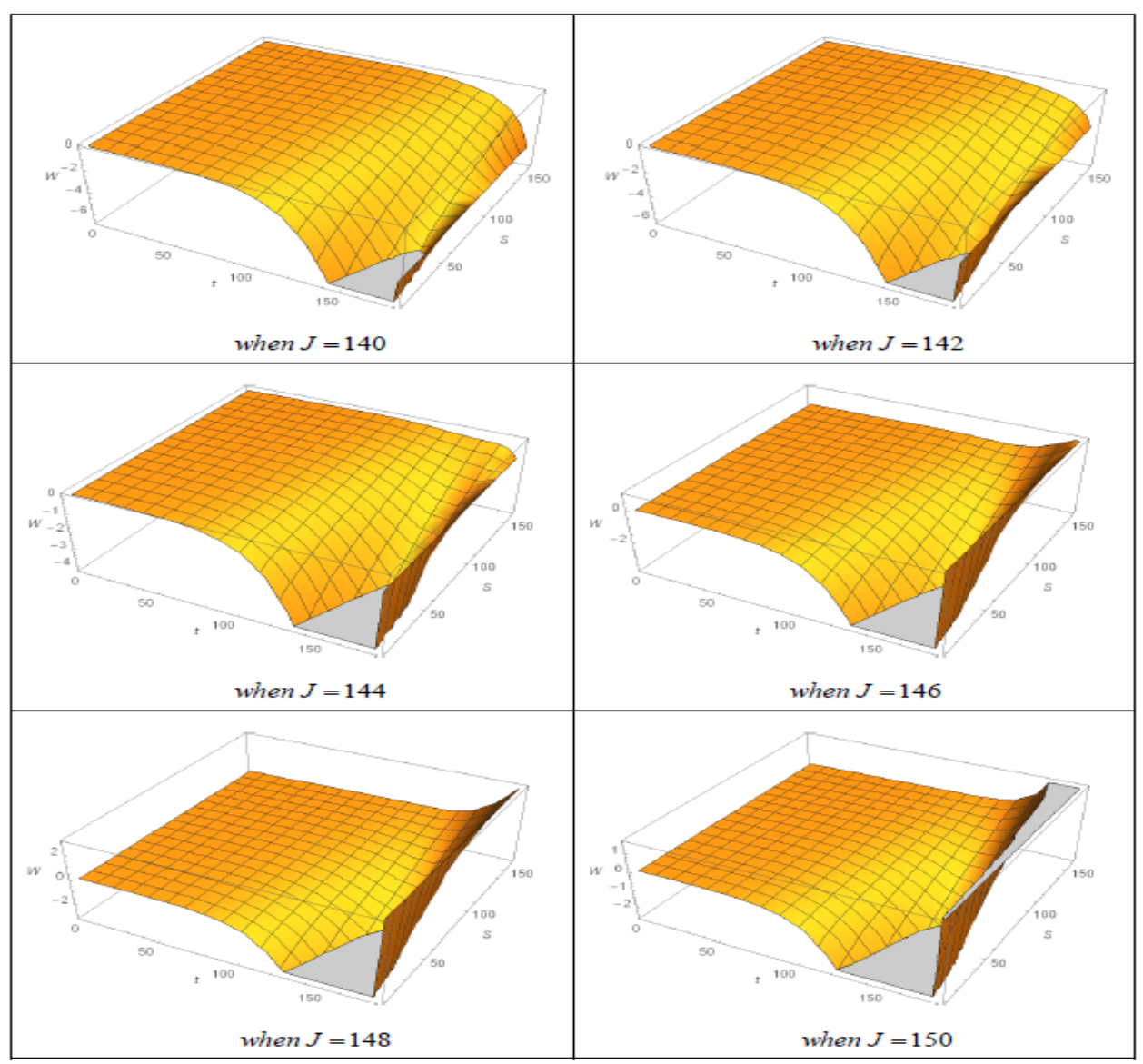

Figure 3. Plot of $\mathrm{w}(\mathrm{S}, \mathrm{J}, \mathrm{t})$ when $S_{0}=K=\$ 145, r=6, q=3$ and $\sigma=29.5$

under the suitable transformations, the function $u$ satisfies the Cauchy problem [17] in the domain $\left\{(x, y) \in \mathbb{R}^{2}, 0 \leq t \leq T\right\}:$

$$
\begin{gathered}
\frac{\partial u}{\partial t}=r W-\left(r-q-\frac{\sigma^{2}}{2}\right)\left[\frac{T-t}{T} \frac{\partial u}{\partial y}+\frac{\partial u}{\partial x}\right]-\frac{\sigma^{2}}{2} \frac{\partial^{2} u}{\partial x^{2}} \\
-\frac{\sigma^{2}}{2}\left(\frac{T-t}{T}\right)^{2} \frac{\partial^{2} u}{\partial y^{2}}-\sigma^{2}\left(\frac{T-t}{T}\right) \frac{\partial^{2} u}{\partial x \partial y} \\
\left.u\right|_{t=T}=e^{x}-e^{y} .
\end{gathered}
$$

Taking the change of variable $\tau=T-t$, we have

$$
\begin{gathered}
\frac{\partial u}{\partial \tau}=\frac{\sigma^{2}}{2}\left(\frac{\tau}{T}\right)^{2} \frac{\partial^{2} u}{\partial y^{2}}+\sigma^{2}\left(\frac{\tau}{T}\right) \frac{\partial^{2} u}{\partial x \partial y}+\frac{\sigma^{2}}{2} \frac{\partial^{2} u}{\partial x^{2}} \\
+\left(r-q-\frac{\sigma^{2}}{2}\right)\left[\frac{\tau}{T} \frac{\partial u}{\partial y}+\frac{\partial u}{\partial x}\right]-r u \\
\left.u\right|_{\tau=0}=e^{x}-e^{y}
\end{gathered}
$$

where

$$
u=u(x, y, \tau)
$$


Using the RDTM to Eq. (3.12), we get the following iterative formula

$$
\begin{aligned}
(m+1) U_{m+1}(x, y)= & \frac{\sigma^{2}}{2 T^{2}} \frac{\partial^{2}}{\partial y^{2}} U_{m-2}(x, y)+\frac{\sigma^{2}}{T} \frac{\partial^{2}}{\partial x \partial y} U_{m-1}(x, y)+\frac{\sigma^{2}}{2} \frac{\partial^{2}}{\partial x^{2}} U_{m}(x, y)+ \\
& \left(r-q-\frac{\sigma^{2}}{2}\right)\left[\frac{1}{T} \frac{\partial}{\partial y} U_{m-1}(x, y)+\frac{\partial}{\partial x} U_{m}(x, y)\right]-r U_{m}(x, y)
\end{aligned}
$$

with the initial condition

$$
u(x, y, 0)=U_{0}(x, y)=e^{x}-e^{y}
$$

For $m=0$

$$
U_{1}(x, y)=0+0+\frac{\sigma^{2}}{2} \frac{\partial^{2}}{\partial x^{2}} U_{0}(x, y)+\left(r-q-\frac{\sigma^{2}}{2}\right)\left[0+\frac{\partial}{\partial x} U_{0}(x, y)\right]-r U_{0}(x, y)
$$

Thus

$$
U_{1}(x, y)=-q e^{x}+r e^{y}
$$

For $m=1$

$$
\begin{aligned}
2 U_{2}(x, y)= & 0+\frac{\sigma^{2}}{T} \frac{\partial^{2}}{\partial x \partial y} U_{0}(x, y)+\frac{\sigma^{2}}{2} \frac{\partial^{2}}{\partial x^{2}} U_{1}(x, y)+ \\
& \left(r-q-\frac{\sigma^{2}}{2}\right)\left[\frac{1}{T} \frac{\partial}{\partial y} U_{0}(x, y)+\frac{\partial}{\partial x} U_{1}(x, y)\right]-r U_{1}(x, y)
\end{aligned}
$$

So

$$
U_{2}(x, y)=\frac{1}{2 !}\left\{q^{2} e^{x}-e^{y}\left(\frac{r-q-\frac{\sigma^{2}}{2}}{T}+r^{2}\right)\right\}
$$

For $m=2$

$$
\begin{aligned}
3 U_{3}(x, y)= & \frac{\sigma^{2}}{2 T^{2}} \frac{\partial^{2}}{\partial y^{2}} U_{0}(x, y)+\frac{\sigma^{2}}{T} \frac{\partial^{2}}{\partial x \partial y} U_{1}(x, y)+\frac{\sigma^{2}}{2} \frac{\partial^{2}}{\partial x^{2}} U_{2}(x, y)+ \\
& \left(r-q-\frac{\sigma^{2}}{2}\right)\left[\frac{1}{T} \frac{\partial}{\partial y} U_{1}(x, y)+\frac{\partial}{\partial x} U_{2}(x, y)\right]-r U_{2}(x, y)
\end{aligned}
$$

So

$$
U_{3}(x, y)=\frac{1}{3 !}\left\{-q^{3} e^{x}-e^{y}\left(\frac{\sigma^{2}}{T^{2}}-3 \frac{r-q-\frac{\sigma^{2}}{2}}{T} r-r^{3}\right)\right\}
$$

For $m=3$

$$
\begin{aligned}
4 U_{4}(x, y)= & \frac{\sigma^{2}}{2 T^{2}} \frac{\partial^{2}}{\partial y^{2}} U_{1}(x, y)+\frac{\sigma^{2}}{T} \frac{\partial^{2}}{\partial x \partial y} U_{2}(x, y)+\frac{\sigma^{2}}{2} \frac{\partial^{2}}{\partial x^{2}} U_{3}(x, y)+ \\
& \left(r-q-\frac{\sigma^{2}}{2}\right)\left[\frac{1}{T} \frac{\partial}{\partial y} U_{2}(x, y)+\frac{\partial}{\partial x} U_{3}(x, y)\right]-r U_{3}(x, y)
\end{aligned}
$$

we have

$$
U_{4}(x, y)=\frac{1}{4 !}\left\{q^{4} e^{x}+4 \frac{\sigma^{2}}{T^{2}} r e^{y}-6\left(\frac{r-q-\frac{\sigma^{2}}{2}}{T}\right) r^{2} e^{y}-3\left(\frac{r-q-\frac{\sigma^{2}}{2}}{T}\right)^{2} e^{y}-r^{4} e^{y}\right\}
$$


For the solution, inverse differential transform of $U_{m}(x, y)$ is defined as below:

$$
\begin{gathered}
u(x, y, \tau)=\sum_{m=0}^{\infty} U_{m}(x, y) \tau^{m} \\
u(x, y, \tau)=e^{x} e^{-q \tau}-e^{y} e^{\left(\frac{\sigma^{2} \tau^{3}}{6 T^{2}}+\frac{\left(r-q-\frac{\sigma^{2}}{2}\right) \tau^{2}}{2 T}-r \tau\right)} \\
\because x=\ln S \Rightarrow e^{x}=S
\end{gathered}
$$

and

$$
y=\frac{t \ln J+(T-t) \ln S}{T} \Rightarrow e^{y}=J^{\frac{t}{T}} S^{\frac{T-t}{T}}
$$

also

$$
\begin{gathered}
\tau=T-t \\
u(x, y, t)=S e^{-q(T-t)}-J^{\frac{t}{T}} S^{\frac{T-t}{T}} e^{\left[\frac{\sigma^{2}(T-t)^{3}}{6 T^{2}}+\frac{\left(r-q-\frac{\sigma^{2}}{2}\right)(T-t)^{2}}{2 T}-r(T-t)\right]}
\end{gathered}
$$

Thus we reached to the exact solution, in closed form

$$
C(S, J, t)-P(S, J, t)=S e^{-q(T-t)}-J^{\frac{t}{T}} S^{\frac{T-t}{T}} e^{\frac{\sigma^{2}(T-t)^{3}}{6 T^{2}}+\frac{\left(r-q-\frac{\sigma^{2}}{2}\right)(T-t)^{2}}{2 T}-r(T-t)}
$$

which is the same solution as obtained in [17]. Consider a six-month call option on stock. If $S_{0}=K=$ $\$ 145, r=6, q=3$ and $\sigma=29.5$ then graphs of $W(S, J, t)$, for average stock price $J$ ranging from $\$ 140$ to $\$ 150$, can be found in Figure 4 on next page. 


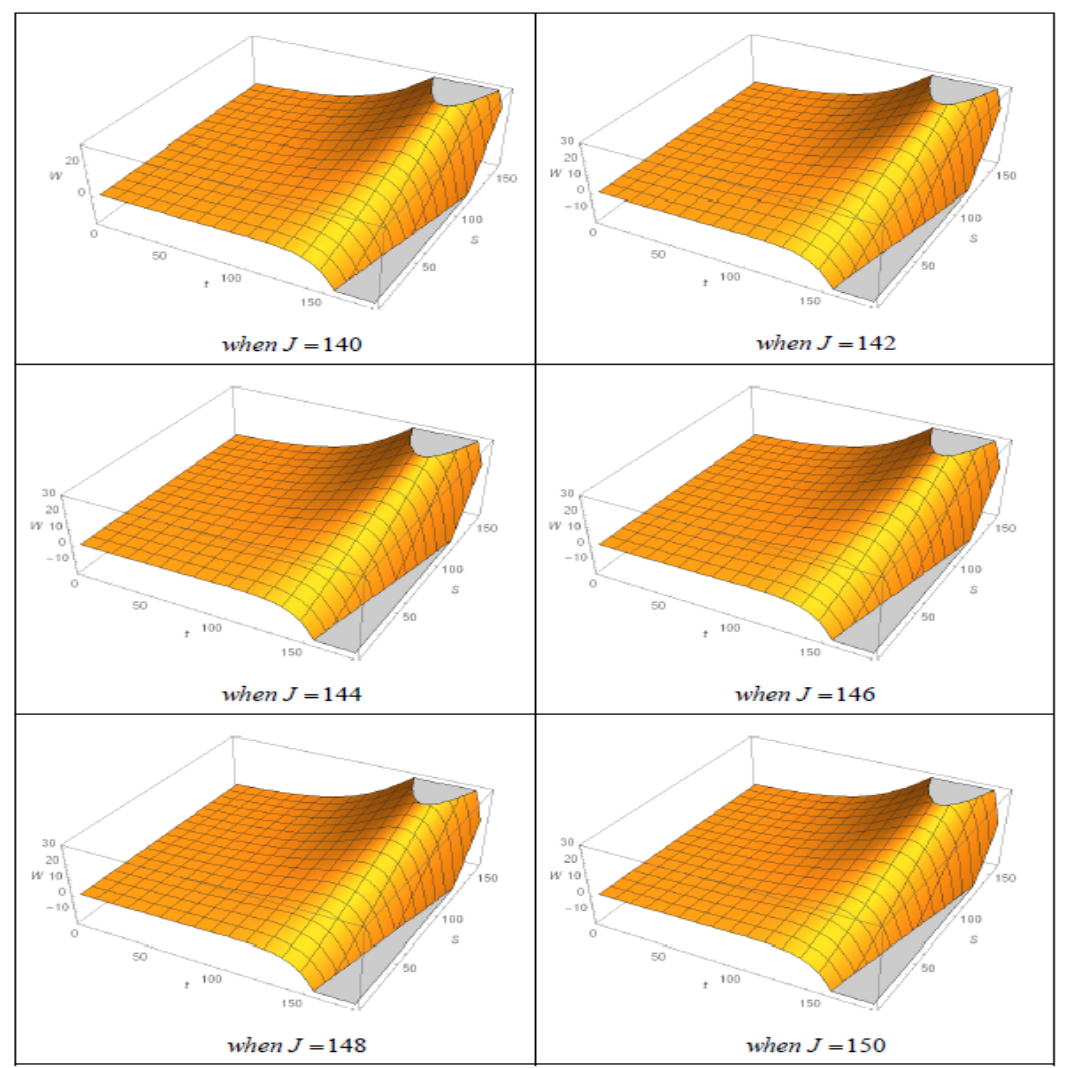

Figure 4. Plot of $\mathrm{w}(\mathrm{S}, \mathrm{J}, \mathrm{t})$ when $S_{0}=K=\$ 145, r=6, q=3$ and $\sigma=29.5$

\section{Conclusion}

The Reduced Differential Transform Method (RDTM) for put-call parities PDEs of Asian options has been presented. The solutions obtained by the method are an infinite power series for appropriate initial condition, which can in turn in a closed form, the exact solution. The efficiency of the presented method is validated through all four versions of PDEs of Asian options put-call parities and found exact solutions same as Lishang solutions. We notice that the RDTM technique is highly accurate, rapidly convergent. RDTM is a very easily implementable mathematical tool for the PDEs in Mathematical Finance subject to appropriate initial condition.

Availability of Data and Materials: This paper contains no any studies with human participants or animals performed by any of the authors. All the data related to the current study were provided along with this paper.

Authors' Contributions: Both authors contributed equally to the manuscript and typed, read, and approved the final manuscript.

Conflicts of Interest: The author(s) declare that there are no conflicts of interest regarding the publication of this paper. 


\section{REFERENCES}

[1] A. Haghbin, S. Hesam, Reduced Differential Transform Method For Solving Seventh Order Sawada-Kotera Equations, J. Math. Computer Sci. 5 (1) (2012), 53-59.

[2] B. Alziary, J. P. Drcamps and P. F. Koehl, A PDE Approach to Asian Options: Analytical and Numerical Evidence, J. Bank. Finance, 21 (1997), 613-640.

[3] Birol İBIŞ, Application of Reduced Differential Transformation Method for Solving Fourth-Order Parabolic Partial Differential Equations, J. Math. Computer Sci. 12 (2014), 124-131.

[4] B. K. Singh, Fractional Reduced Differential Transform Method for Numerical Computation of a System of Linear and Nonlinear Fractional PDEs, Int. J. Open Probl. Compt. Math. 9 (3) (2016), 20-38.

[5] C. Fan, P. Li, Y. Xue, Application of Differential Equations in Enzyme Kinetics, Chem. Eng. Trans. 71 (2018), 883-888.

[6] D.I. Cruz and J.M. Gonzalez., A Different Approach for Pricing Asian Options, Appl. Math. Lett. 21 (2008), 303-306.

[7] E. Barucci, S. Polidoro and V. Vespri, Some Results on Partial Differential Equations and Asian Options, Math. Models Methods Appl. Sci. 11 (3) (2001), 475-497.

[8] H. Jafari, H. Jassim, S. Moshokoa, V. Ariyan and F. Tchier, Reduced Differential Transform Method for Partial Differential Equations Within Local Fractional Derivative Operators, Adv. Mech. Eng. 8 (4) (2016), 1-6.

[9] H. Geman and M. Yor, Bessel Processes, Asian options, and Perpetuities, Math. Finance, 3 (4) (1993), 349-375.

[10] H. Rouhparvar, Computational Technique of Linear Partial Differential Equations by Reduced Differential Transform Method, Int. J. Ind. Math. 8 (4) (2016), Article ID IJIM-00651.

[11] J. N. dewynne and W. T. Shaw, Differential Equations and Asymptotic solutions for Arithmetic Asian Options: BlackScholes Formulae for Asian Rate Calls, Eur. J. Appl. Math. 19 (4) (2008), 353-391.

[12] Y. Keskin, Ph.D Thesis, Selcuk University, 2010.

[13] Y. Keskin, G. Oturance, Reduced Differential Transform Method for Partial Differential Equations, Int. J. Nonlinear Sci. Numer. Simul. 10 (6) (2009), 741-749.

[14] Y. Keskin, G. Oturance, Reduced Differential Research Transform Method for Fractional Partial Differential Equations, Nonlinear Sci. Lett. A, 1 (1) (2010), 61-72.

[15] Y. Keskin, G. Oturance, Application of Reduced Differential Transformation Method for Solving Gas Dynamics Equation, Int. J. Contemp. Math. Sci. 5 (22) (2010), 1091-1096.

[16] Y.K. Kwok, Mathematical Models of Financial derivatives, 2nd Ed., Springer, Berlin, 2008.

[17] J. Lishang, Mathematical Modeling and Methods of Option Pricing, World Scientific, Singapore, 2005.

[18] Mahmoud. R, Using the Reduced Differential Transform Method to Solve Nonlinear PDEs Arises in Biology and Physics, World Appl. Sci. J. 23 (8) (2013), 1037-1043.

[19] Mohammad. T., Vineet K., Srivastava: A Computational Modelling of Micro Strip Patch Antenna and Its Solution By RDTM, Alex. Eng. J. 57 (2018), 1877-1881.

[20] L. C. G. Rogers and Z. Shi, The Value of an Asian Option, J. Appl. Prob. 32 (4) (1995), 1077-1088.

[21] V. Srivastava, M. Awasthi, M. Tamsir, RDTM Solution of Caputo Time Fractional-Order Hyperbolic Telegraph Equation, AIP Adv. 3 (3) (2013), 032142.

[22] S. Servi, Y. Keskin, G. Oturanç, Reduced Differential Transform Method for Improved Boussinesq Equation, AIP Conf. Proc. 1648 (2015), 370012 .

[23] T.R. Ramesh Rao, Numerical Solution of Sine Gordon Equations Through Reduced Differential Transform Method, Glob. J. Pure Appl. Math. 13 (7) (2017), 3879-3888. 
[24] Z. Ali, R. Rozita and R. Hazli, New Pricing Formula for Arithmetic Asian Options using PDE Approach, App. Math. Sci. 5 (77) (2011), 3801-3809.

[25] Z. Ali and R. Rozita, Solving an Asian option PDE via the Laplace Transform, ScienceAsia 39S (2013), 67-69. 